\title{
Development of novel microsatellite markers to analyze the genetic structure of dog populations in Taiwan
}

\author{
Fang-Yu Lai ${ }^{1}$, Yu-Chen Lin ${ }^{1}$, Shih-Torng Ding ${ }^{1}$, Chi-Sheng Chang ${ }^{2}$, Wi-Lin Chao ${ }^{3}$, and Pei-Hwa Wang ${ }^{1, *}$
}

\section{* Corresponding Author: Pei-Hwa Wang \\ Tel: +886-02-33664164, \\ Fax: +886-02-23724070 \\ E-mail: demonwang@ntu.edu.tw}

${ }^{1}$ Key Laboratory of Animal Genetics,

Breeding and Bioresources, Department of Animal Science and Technology, College

of Bioresources and Agriculture, National

Taiwan University, Taipei 10672, Taiwan

2 Department of Animal Science, Chinese

Culture University, Taipei 11114, Taiwan

${ }^{3}$ Department of Animal Industry, Council of

Agriculture, Taipei 100212., Taiwan

ORCID

Fang-Yu Lai

https://orcid.org/0000-0001-9912-6647

Shih-Torng Ding

https://orcid.org/0000-0002-9866-1776

Chi-Sheng Chang

https://orcid.org/0000-0003-3885-1313

Pei-Hwa Wang

https://orcid.org/0000-0003-4405-3166

Submitted Nov 26, 2021; Revised Jan 26, 2022; Accepted Feb 16, 2022
Objective: Alongside the rise of animal-protection awareness in Taiwan, the public has been paying more attention to dog genetic deficiencies due to inbreeding in the pet market. The goal of this study was to isolate novel microsatellite markers for monitoring the genetic structure of domestic dog populations in Taiwan.

Methods: A total of 113 DNA samples from three dog breeds-beagles (BEs), bichons (BIs), and schnauzers (SCs) - were used in subsequent polymorphic tests applying the 14 novel microsatellite markers that were isolated in this study.

Results: The results showed that the high level of genetic diversity observed in these novel microsatellite markers provided strong discriminatory power. The estimated probability of identity $\left(\mathrm{P}_{(\mathrm{ID})}\right)$ and the probability of identity among sibs $\left(\mathrm{P}_{(\mathrm{ID}) \text { sib }}\right)$ for the 14 novel microsatellite markers were $1.7 \times 10^{-12}$ and $1.6 \times 10^{-5}$, respectively. Furthermore, the power of exclusion for the 14 novel microsatellite markers was $99.98 \%$. The neighbor-joining trees constructed among the three breeds indicated that the 14 sets of novel microsatellite markers were sufficient to correctly cluster the BEs, BIs, and SCs. The principal coordinate analysis plot showed that the dogs could be accurately separated by these 14 loci based on different breeds; moreover, the Beagles from different sources were also distinguished. The first, the second, and the third principal coordinates could be used to explain $44.15 \%, 26.35 \%$, and $19.97 \%$ of the genetic variation.

Conclusion: The results of this study could enable powerful monitoring of the genetic structure of domestic dog populations in Taiwan.

Keywords: Dog; Novel Microsatellite Marker; Population Genetic Analysis

\section{INTRODUCTION}

After strong artificial selection for over 150 years, morphological variation has been created in dog populations and more than 400 canine breeds are currently registered worldwide by the Fédération Cynologique Internationale (FCI), and other federations such as the American and British Kennel Clubs [1]. With over 470 million dogs being kept as companion animals worldwide, they were ranked as the leading type of pet in 2018 [2]. In Taiwan, according to survey statistics from the Council of Agriculture, the number of dogs being bred by the end of 2017 had reached 1.78 million. The overall sales revenue of petrelated industries has increased from 15.5 billion New Taiwan dollars (NT\$) in the past 10 years to 26.6 billion NT\$ (Statistical Bulletin, Ministry of Finance, Republic of China; http://service.mof.gov.tw/File/Attach/86088/File_21588.pdf). The previous roles of companion animals, such as hunting, security, and assistance, have gradually shifted towards them being regarded as family members. With the improvement of the status of pets, owners are increasingly paying attention to their quality of life. Therefore, the demand for various goods and services aimed at companion animals is also increasing. In the early stage of 
the Taiwan dog-sales market, the characteristics prioritised included the ability to guard, search, attack, and hunt; more recently, these ideals have been superseded by the need to provide companionship and prestige, which have made a recognized pedigree a major factor for buyers. In Taiwan, some legal purebreed grounds used closed flock breeding to keep their dog broodlines, including those three breeds in this study. Especially the Beagles, which have been used to hunt hares in the British Isles for centuries, and which were brought to the United States in 1880 to breed in large numbers. The modern Beagles have been modified to become a pet dog, and were often used as experimental dogs [3]. It has considerable medical research value, and this dog was also cultivated as laboratory animal for medical research in Taiwan.

So far, many dog breeds have been developed in order to meet appearance standards and maintain the purity of their bloodlines. Breeding companies usually adopt inbreeding methods, which can lead to the occurrence of many genetic diseases. Generally, in the natural state-unlike with experimental animals for which it can be essential to reduce individual differences for study purposes-inbreeding approaches have seldom been used, in order to avoid inbreeding depression [4]. The Royal Society for the Prevention of Cruelty to Animals (RSPCA) pointed out that dogs are now subject to more than 300 genetic diseases. Not only do the animals have to bear great suffering, but their owners also experience mental pressures and financial losses [5]. Therefore, in order to maintain high heterozygosity and stability of the genetic background of the entire population, it is necessary to have a reliable breeding system and genetic monitoring [6]. However, in Taiwan, the genealogical and registration data requirements for many breeds of dog are incomplete or remain to be established, and most of the certificates of pedigree produced by breeding sites in the market lack the backing of publicly trusted authorities, so trading disputes arise from time to time. Although trading in companion animals is discouraged in many countries today, the market in Taiwan is booming. In order to prevent companion animals from being afflicted by genetic diseases, in addition to promoting care by and education of owners, an important factor is reducing inbreeding. The Kennel Club has established a breeding certification system for 68 breeds since 2008, with the total number now reaching 222 breeds. Breeders can inquire about the diseases to be screened for each dog and the procedures for obtaining certification. After the puppies are certified by the Kennel Club, the breeders are issued with a puppy sale wallet; this measure not only protects the profits of the owner, but also reduces disputes over the sale of companion animals [7].

Regarding the research on dog microsatellite markers, a lot of information has been discovered in conjunction with the elucidation of DNA sequences [8]. As early as the 1990s, there have been many studies on dog microsatellite markers $[9,10]$. The application of canine microsatellite markers in modern times has mainly focused on two fields: one is evaluating the genetic structure polymorphism of populations, and the other is proving a platform for individual identification or paternity [11,12]. Wictum et al [13] searched for published dog genome sequences, and selected suitable microsatellite markers based on the stability and high polymorphism that were required for forensic applications. A total of 15 microsatellite markers and a marker related to gender comprise the multiplex system, DogFiler [13]. This is the first dog-identification data system created based on the recommendations of the Scientific Working Group for DNA Analysis Methods (SWGDAM) in the United States. At present, DogFiler has been integrated into forensic casework, and is widely accepted by courts in the United States. Owing to the relatively long period of strong artificial selection, the differentiation between dog breeds has been large. The sequences on both sides of microsatellite markers may have different degrees of variation, making it impossible to perform polymerase chain reaction (PCR) amplification in similar breeds. Even if the amplification is successful, the number of alleles and polymorphisms may be far lower than in the original breeds [14]. Therefore, when conducting research on the population genetics of specific breeds of dog, it is necessary to develop new microsatellite markers.

To date, only a few domestic biotechnology companies in Taiwan use foreign dog microsatellite commercial kits, such as the StockMarks for dogs genotyping kit, for individual genetic analysis or genetic structure analysis at dog breeding sites. So, are they applicable to Taiwan? To our knowledge, there are no relevant published reports on existing dog breeds for reference and analysis. Therefore, the development of microsatellite markers suitable for the companion animal population in Taiwan is a crucial task to establish a moleculardetection platform for domestic dogs. Moreover, such a platform will make it possible to evaluate the inbreeding level of the dog population in Taiwan. In addition to assisting with the formulation of domestic dog breeding-management policies, this could also enhance Taiwan's positive governance perspectives on animal protection and animal welfare.

\section{MATERIALS AND METHODS}

\section{Experimental animals and sample collection}

In total, five populations of dogs from legal breeding ground were investigated in this study. Blood samples were collected from 113 individuals belonging to these three populations which were popular in Taiwan: 17 bichons (BIs); 14 schnauzers (SCs); 74 Taiwanese beagles (BETs); and eight Japanese beagles (BEJs), two of which were distinguished as group A (BEJAs) and six of which were distinguished as group B 
(BEJBs) (Table 1). For each dog, $5 \mathrm{~mL}$ of blood was drawn from the jugular or cephalic vein. Whole genomic DNA (gDNA) was then extracted with the Genomic DNA Isolation Reagent (GenePure Technology Co., Ltd, Taichung, Taiwan) using the standard phenol-chloroform method. NanoDrop 2000c (Thermo Fisher Scientific Inc., Waltham, MA, USA) detected the DNA concentration and the optical density (OD) 260/280 values between 1.8 and 2.0, and samples were stored in a refrigerator at $-20^{\circ} \mathrm{C}$ for later use. All animal experiments were approved by the Institutional Animal Care and Use Committee of our university (Protocol number: NTU Animal Experiments No. 2012-089).

\section{Isolation of microsatellite loci}

The gDNA was isolated from blood samples taken from one male and one female BI using the following steps, which were slightly modified from the procedure described by Glenn and Schable [15]. The isolated gDNA was partially digested with RsaI and XmnI (NEB, Ipswich, MA, USA) until most of the DNA fragments were between 300 and 1,000 base pairs (bp) in length. Re-naturing of single-strand SuperSNX24 forward (5'GTTTAAGGCCTAGCTAGCAGAATC3') and SuperSNX24+4P reverse (5'pGATTCTGCTAGCTAGGC CTTAAACAAAA3') formed SuperSNX24 linkers that were ligated to the digested DNA fragments. Linked DNA fragments were then amplified with SuperSNX24. Next, microsatellite markers including fragments with biotinated probes were isolated and enriched. The following three probe compositions were used: i) $(\mathrm{TG})_{12},(\mathrm{ACT})_{12},(\mathrm{ACTG})_{6}$, and $(\mathrm{ACAG})_{6}$; ii) $(\mathrm{AG})_{12},(\mathrm{ACAT})_{8},(\mathrm{AACT})_{8}$, and $(\mathrm{AAGT})_{8}$; and iii $(\mathrm{AAG})_{8}$, $(\mathrm{AAAC})_{6},(\mathrm{AATC})_{6}$, and $(\mathrm{AGAT})_{6}$. The biotinated probes were then annealed to fragments of gDNA containing complementary regions. Finally, the microsatellite-containing fragments were enriched using streptavidin-labeled metal beads (Dynabeads M-280 Streptavidin, catalog \#11205D; Invitrogen, Waltham, MA, USA). The enriched segments were then cloned into pGEM-T Easy vector (pGEM-T Easy Vector system; Promega, Madison, WI, USA) and sequenced with an ABI 3730XL DNA Analyzer (Applied Biosystem PRISM, Waltham, MA, USA). The fragments with higher

Table 1. Number of dogs of the three breeds from different farms

\begin{tabular}{lccc}
\hline Breed & Abbreviation $^{\text {1) }}$ & Farm $^{2)}$ & Sample size \\
\hline Bichon & $\mathrm{BI}$ & $\mathrm{K}$ & 17 \\
Schnauzer & $\mathrm{SC}$ & $\mathrm{K}$ & 14 \\
Beagle & $\mathrm{BET}$ & $\mathrm{P}$ & 74 \\
& BEJA & $\mathrm{P}$ & 2 \\
& BEJB & NTU & 6 \\
Total & & & 113 \\
\hline
\end{tabular}

1) BET, beagle bred in Taiwan; BEJA and BEJB, beagle imported from Japan.

2) $\mathrm{K}$ farm located in Kaohsiung city in Taiwan; $\mathrm{P}$ farm located in Pingtung county in Taiwan; NTU, National Taiwan University. numbers of repeats were selected for polymorphism testing.

\section{Polymerase chain reaction and polymorphism testing}

The selected highly repeated fragments were subjected to PCR and polymorphism testing, which would verify whether the microsatellite loci could be amplified and used to show diversity in the investigated populations. Primers for loci amplification were designed using Primer3plus. CAG-tag (5'-CAGTCGGGCGTCATCA-3') or M13Reverse (5'-GGAA ACAGCTATGACCAT- $3^{\prime}$ ) was added to the $5^{\prime}$ end of one of each primer pair. Following the protocol described by Schuelke [16], a fluorescent dye-labeled tag, as a third primer, was used with the primer pair to amplify the target fragments that were detectable upon capillary electrophoresis. Eight dog samples from four BIs and four SCs were tested at a $20-\mu \mathrm{L}$ volume using a thermalcycler (GeneAmp PCR system 9700; Applied Biosystems, USA) containing 0.5 U Taq DNA polymerase (TAKARA, Kusatsu, Shiga, Japan), $1 \times$ PCR buffer (1.5 mM $\mathrm{MgCl}_{2}$ ), $0.2 \mathrm{mM}$ deoxyribose nucleotide triphosphate (dNTP), $0.2 \mu \mathrm{M}$ unlabeled primer, $0.04 \mu \mathrm{M}$ tag-labeled primer, 0.16 $\mu \mathrm{M}$ dye-labeled tag, and $50 \mathrm{ng}$ gDNA. The PCR cycling program was as follows: $95^{\circ} \mathrm{C}$ for $5 \mathrm{~min}, 35$ cycles of $95^{\circ} \mathrm{C}$ for 30 s, $50^{\circ} \mathrm{C}$ to $65^{\circ} \mathrm{C}$ for $40 \mathrm{~s}, 72^{\circ} \mathrm{C}$ for $40 \mathrm{~s}$, and a final elongation at $72^{\circ} \mathrm{C}$ for $7 \mathrm{~min}$. The amplified microsatellite PCR products were analyzed with a DNA analyzer (ABI PRISM 3730 DNA analyzer; Applied Biosystems, USA). Allelic sizes of all loci were estimated relative to the in-line GeneScan500 LIZ Size Standard marker (ABI PRISM; Applied Biosystems, USA). The fragment size was calibrated and analyzed with Peak Scanner Software version 1.0 (ABI PRISM; Applied Biosystems, USA). Those loci that had an allele number greater than two and similar annealing temperatures were selected for whole-population analysis.

\section{Multiplex polymerase chain reaction}

In total, 14 microsatellite loci were selected (Table 2). The $5^{\prime}$ end of each forward primer was labeled with the fluorescent dyes fluorescein (FAM), 2'-chloro-7'phenyl-1,4-dichloro-6carboxy-fluorescein (VIC), red color fluorescent (PET), or 2'-chloro-5'-fluoro-7', 8'-benzo-1,4-dichloro-6-carboxyfluorescein (NED). Multiplex PCR was performed on a $30-\mu \mathrm{L}$ reaction containing $1 \mathrm{U}$ Taq DNA polymerase (TAKARA, Japan), $1 \times$ PCR buffer $(1.5 \mathrm{mM} \mathrm{MgCl}$ ), $0.3 \mathrm{mM}$ dNTP, 0.3 $\mu \mathrm{M}$ forward and reverse primer, and a 100-ng DNA template. The steps of the PCR program and genotype detection were as described above.

\section{Statistical analysis}

For each locus and population, and across populations, commonly derived statistics from the microsatellite genotypic data, including allele frequencies, the observed number of alleles (No), the observed heterozygosity (Ho), the expected 
Table 2. Primer sequences, repeated motifs, and fluorescent labeling of 14 microsatellite loci

\begin{tabular}{|c|c|c|c|c|}
\hline Locus & Primer sequence $\left(5^{\prime}-3^{\prime}\right)$ & Repeat motif & Size (bp) & Label \\
\hline SEL005 & $\begin{array}{l}\text { F: AGCTTGCCAACTTCACTCGT } \\
\text { R: TCCTGCTTGGTCTCCTGATT }\end{array}$ & $(\mathrm{CTTTT})_{17}$ & $186-260$ & FAM \\
\hline SEL105 & $\begin{array}{l}\text { F: TCAACAATTGAAATATACAAGTTAGCA } \\
\text { R: GGGCATGATCCTAGAGTCCA }\end{array}$ & $(\mathrm{GA})_{10}$ & $166-172$ & FAM \\
\hline SEL117 & $\begin{array}{l}\text { F: CAGCCTGGAGATACACAGCA } \\
\text { R: CCACTGGAAACACAGCAGAA }\end{array}$ & $(\mathrm{TG})_{17}$ & $147-153$ & FAM \\
\hline SEL030 & $\begin{array}{l}\text { F: TGGAGACTCGGGATCAAATC } \\
\text { R: CCTACCCATTTCGCTCATGT }\end{array}$ & $(\mathrm{TC})_{18}$ & $189-209$ & NED \\
\hline SEL031 & $\begin{array}{l}\text { F: GACCATCTCCATTGAGAACCA } \\
\text { R: TACAACGGTCTTTCCCAGGT }\end{array}$ & $(\mathrm{CT})_{13}$ & 159-175 & NED \\
\hline SEL068 & $\begin{array}{l}\text { F: CTTGCCCCTGAGCAAGATAC } \\
\text { R: GGTGTGTCCGCCTTAAAGAA }\end{array}$ & $(C A)_{12}$ & $160-201$ & VIC \\
\hline SEL098 & $\begin{array}{l}\text { F: ATACAGTTGGTGCCCAAAAA } \\
\text { R: CTCCCTGCTCACACACACAC }\end{array}$ & $(\mathrm{GA})_{8}(\mathrm{TG})_{10}$ & $226-236$ & VIC \\
\hline SEL034 & $\begin{array}{l}\text { F: GCTTCTCACATGCAACATGG } \\
\text { R: GGCCTCCCAAGAAATGGTAT }\end{array}$ & $(C A)_{11}$ & 187-193 & PET \\
\hline SEL094 & $\begin{array}{l}\text { F: GACCATCTCCAGCCATCCTA } \\
\text { R: TGGGTTTGAATTGGCTAACA }\end{array}$ & $(\mathrm{TC})_{14}$ & 187-191 & FAM \\
\hline SEL115 & $\begin{array}{l}\text { F: TCACAAATGGCAAAATCTTTCTT } \\
\text { R: AAGCAACCCAAGTGTCCATC }\end{array}$ & $(\text { GATA })_{19}(\text { GGTA })_{18}$ & 203-263 & FAM \\
\hline SEL035 & $\begin{array}{l}\text { F: CACTGAGCATCCACTGAAGG } \\
\text { R: CACCCACCATGGCTCTCTAT }\end{array}$ & $(\mathrm{TG})_{15}(\mathrm{AG})_{11}$ & $185-211$ & NED \\
\hline SEL118 & $\begin{array}{l}\text { F: CTGGGCTGGGTAGTCTGTTC } \\
\text { R: TCCCCCAAGTGATTCTTCTG }\end{array}$ & $(\mathrm{CA})_{19}$ & 159-172 & NED \\
\hline SEL025 & $\begin{array}{l}\text { F: GCACAGGCTTTTCATATCCA } \\
\text { R: AATGAGTGAATGGGCACCTC }\end{array}$ & $(\mathrm{CT})_{14}(\mathrm{CA})_{14}$ & $143-157$ & PET \\
\hline SEL093 & $\begin{array}{l}\text { F: GTGGTAGGGAGAGGGACAGA } \\
\text { R: CTCCTGCTGACCTTTCTTGG }\end{array}$ & $(\mathrm{GA})_{19}$ & $174-176$ & PET \\
\hline
\end{tabular}

FAM, blue color fluorescent; NED, yellow color fluorescent; VIC, green color fluorescent; PET, red color fluorescent.

heterozygosity $\left(\mathrm{H}_{\mathrm{E}}\right)$, and the polymorphic information content (PIC), were calculated with the Microsatellite Toolkit. The Hardy-Weinberg equilibrium test was performed using the GENEPOP computer program [17], which also was used to estimate $F$-statistics (the global mean inbreeding coefficient $\left[F_{\mathrm{IT}}\right]$, the average inbreeding coefficient of an individual with respect to the local subpopulation $\left[F_{\mathrm{IS}}\right]$, and the average inbreeding coefficient of subpopulations relative to the total population $\left[F_{\mathrm{ST}}\right]$ ) for each locus, the pairwise $F_{\mathrm{ST}}$ between populations, and the average inbreeding coefficient $\left(F_{\text {IS }}\right)$. Nei's genetic distance $\left(D_{A}\right)$ [18] between populations was measured with the Microsatellite Analyzer [19]. A phylogenetic tree was generated via the PHYLIP program [20] using the neighbor-joining $(\mathrm{NJ})$ method with a bootstrap test of 1,000 resampling of loci with replacement [21].

The model-based approach proposed for the population structure analysis of the dog populations was carried out with the software STRUCTURE 2.3.1 [22], which was used to assess the genomic clustering $(\mathrm{K})$ of the sample. To obtain a representative value of $\mathrm{K}$ for data modeling, 10 independent runs were performed for each value from one to seven. The run length was set to 100,000 burn-ins followed by 100,000 iterations. In addition, a principal coordinate analysis
(PCoA) three-dimensional (3D) map drawn by GenAlEx [23] was used to confirm the inter-population situation.

The effectiveness of individual identification is expressed by the probability of identity $\left(\mathrm{P}_{(\mathrm{ID})}\right)$, which is the probability that two individuals are randomly selected from the population and the genotypes of the two are identical at a single locus. The theoretical expectations are as follows:

$$
\mathrm{P}_{(\mathrm{ID})}=\sum_{i=1}^{n} p_{i}^{4}+4 \sum_{i=1}^{n} \sum_{j=1}^{n-1} p_{i}^{2} p_{j}^{2}
$$

where $p_{i}$ and $p_{j}$ are the frequencies of the ith and jth alleles, and $\mathrm{i} \neq \mathrm{j}$. When the molecular markers are co-dominant, such as microsatellite markers, and the identified individuals have close kinship, the following probability of identity among sibs $\left(\mathrm{P}_{(\mathrm{ID}) \text { sib }}\right)$ is applied:

$$
\mathrm{P}_{(\mathrm{ID}) \mathrm{sib}}=0.25+0.5 \sum_{i=1}^{n} p_{j}^{2}+0.5\left(\sum_{i=1}^{n} p_{j}^{2}\right)^{2}-0.25 \sum_{i=1}^{n} p_{j}^{4}
$$

where $p_{j}$ is the frequency of the $j$ th allele [24]. The calcula- 
tion of the PE is also based on the formula mentioned by Jamieson [25].

\section{RESULTS}

\section{Polymorphism, heterozygosity, and F-Statistics of novel microsatellite loci}

The 14 microsatellite markers were used to perform genotyping on 113 samples from the three dog breeds (SCs, BIs, and BETs/BEJs). Polymorphism was clearly observed at most of the microsatellite loci in the three breeds. The genetic characteristics of the 14 microsatellite loci are listed in Table 3. The average number of alleles per locus $(\mathrm{Na})$ was 6.3. The actual number of alleles ranged from 2 (SEL093 and SEL094) to 13 (SEL115). The average number of effective alleles per locus (Ne) ranged from 1.4 (SEL094) to 7.6 (SEL005), with an average across loci value of 3.6. The PIC value ranged from 0.249 (SEL094) to 0.855 (SEL005), with an overall average value of 0.612 . All of the selected microsatellite loci in this study were sufficiently polymorphic, indicating that they were suitable for the genetic analysis of dogs.

The $\mathrm{H}_{\mathrm{E}}$ among the 14 microsatellite loci ranged from 0.293 (SEL094) to 0.873 (SEL005), with an average of 0.662. The $\mathrm{H}_{\mathrm{O}}$ among the 14 microsatellite loci ranged from 0.248 (SEL094) to 0.814 (SEL005), with an average of 0.567 (Table 3). However, there were eight loci-namely, SEL025, SEL030, SEL031, SEL035, SEL068, SEL098, SEL115, and SEL118-that significantly departed from the Hardy-Weinberg equilibrium $(\mathrm{p}<0.01)$.

The Wright's $F$-statistic values $\left(F_{\mathrm{IS}}, F_{\mathrm{IT}}\right.$, and $\left.F_{\mathrm{ST}}\right)$ for each locus are shown in Table 3. The average $F_{\text {IS }}$ for all the loci was 0.002 , and the $F_{\text {IS }}$ per locus varied from -0.191 (SEL093) to 0.328 (SEL115). The average $F_{\text {IT }}$ for all the loci was 0.209 , and the $F_{\text {IT }}$ per locus varied from -0.070 (SEL034) to 0.506 (SEL115). The mean $F_{\mathrm{ST}}$ for all the loci was 0.212 . This value implied that around $21.2 \%$ of the total genetic variation was caused by population differences and that $78.8 \%$ of the total genetic variation was due to genetic differentiation among the individuals within each population.

\section{Intra-population genetic variability}

The genetic statistics relating to polymorphism, including $\mathrm{H}_{\mathrm{E}}, \mathrm{H}_{\mathrm{O}}$, PIC, the mean observed number of alleles, and the mean effective number of alleles, were calculated to estimate the allelic diversity at each locus of the population. These genetic parameters across the 14 loci for the three dog populations are listed in Table 4. The $\mathrm{H}_{\mathrm{E}}$ varied from 0.480 (SCs) to 0.624 (BEs), whereas the $\mathrm{H}_{\mathrm{O}}$ varied from 0.485 (SCs) to 0.587 (BEs), and the PIC ranged from 0.407 (SCs) to 0.567 (BEs). The SC population had the lowest values of $\mathrm{H}_{\mathrm{O}}, \mathrm{H}_{\mathrm{E}}$, and PIC.

Among the three breeds, the BE population had the highest observed mean number of alleles (MNA) (5.3), followed by the BI (4.0) and SC (3.3) populations, while the latter had the smallest observed MNA. Negative $F_{\text {IS }}$ values were observed in the BI and SC populations, indicating an insufficient degree of inbreeding. The deviation from the Hardy-Weinberg proportions within populations $\left(F_{\text {IS }}\right)$ varied from -0.032 to 0.045. The highest inbreeding effects were found in the BE population (0.045), which significantly deviated from the

Table 3. Genetic variability of the total dog population from three dog breeds genotyped with 14 sets of novel microsatellite markers

\begin{tabular}{|c|c|c|c|c|c|c|c|c|c|}
\hline Locus & $F_{\mathrm{IS}}{ }^{1)}$ & $F_{\mathrm{ST}}^{1)}$ & $F_{\mathrm{IT}}{ }^{1)}$ & $\mathbf{N}{ }^{1)}$ & $\mathbf{N}_{\mathrm{e}}{ }^{1)}$ & $\mathbf{H}_{0}{ }^{1)}$ & $\mathrm{H}_{\mathrm{E}}{ }^{1)}$ & $\mathrm{PIC}^{1)}$ & Exact test of $\mathrm{HWE}^{2}$ \\
\hline SEL005 & 0.001 & 0.119 & 0.119 & 10 & 7.6 & 0.814 & 0.873 & 0.855 & NS \\
\hline SEL025 & -0.013 & 0.244 & 0.234 & 8 & 4.4 & 0.670 & 0.775 & 0.741 & * \\
\hline SEL030 & 0.092 & 0.249 & 0.318 & 9 & 4.5 & 0.602 & 0.779 & 0.741 & * \\
\hline SEL031 & 0.135 & 0.340 & 0.429 & 5 & 3.5 & 0.487 & 0.717 & 0.667 & * \\
\hline SEL034 & -0.091 & 0.019 & -0.070 & 3 & 2.2 & 0.602 & 0.557 & 0.489 & NS \\
\hline SEL035 & 0.002 & 0.295 & 0.297 & 10 & 5.2 & 0.664 & 0.813 & 0.791 & * \\
\hline SEL068 & -0.104 & 0.161 & 0.073 & 7 & 2.9 & 0.655 & 0.654 & 0.608 & * \\
\hline SEL093 & -0.191 & 0.138 & -0.027 & 2 & 1.7 & 0.451 & 0.412 & 0.326 & NS \\
\hline SEL094 & -0.010 & 0.266 & 0.258 & 2 & 1.4 & 0.248 & 0.293 & 0.249 & NS \\
\hline SEL098 & -0.116 & 0.355 & 0.281 & 6 & 3.8 & 0.637 & 0.739 & 0.695 & * \\
\hline SEL105 & -0.041 & 0.078 & 0.040 & 4 & 2.4 & 0.584 & 0.587 & 0.494 & NS \\
\hline SEL115 & 0.328 & 0.265 & 0.506 & 13 & 3.8 & 0.416 & 0.737 & 0.714 & * \\
\hline SEL117 & -0.034 & 0.132 & 0.103 & 3 & 2.3 & 0.549 & 0.574 & 0.481 & NS \\
\hline SEL118 & 0.077 & 0.306 & 0.359 & 6 & 4.1 & 0.566 & 0.757 & 0.714 & * \\
\hline Mean & 0.002 & 0.212 & 0.209 & 6.3 & 3.6 & 0.567 & 0.662 & 0.612 & \\
\hline
\end{tabular}

${ }^{1)} F_{I S}$, Wright's F-statistics, within subpopulation inbreeding estimate; $F_{S T}$, Wright's F-statistics, among subpopulation differentiation estimate; $F_{I T}$, Wright's F-statistics, within total population inbreeding estimate; $\mathrm{N}_{a}$, number of alleles; $\mathrm{N}_{e}$, effective number of alleles; $\mathrm{H}_{0}$, observed heterozygosity; $\mathrm{H}_{\mathrm{E}}$, expected heterozygosity; PIC, polymorphism information content.

2) Hardy-Weinberg equilibrium.

* Significant $(p<0.01)$ departure from the Hardy-Weinberg equilibrium.

NS, not significant. 
Table 4. Genetic parameters across 14 loci in the three dog populations

\begin{tabular}{|c|c|c|c|c|c|c|c|c|}
\hline \multirow{2}{*}{ Breed } & \multirow{2}{*}{ Sample size } & \multicolumn{2}{|c|}{ MNA } & \multicolumn{2}{|c|}{ Mean heterozygosity } & \multirow{2}{*}{ PIC } & \multirow{2}{*}{ FIS } & \multirow{2}{*}{ HWE test } \\
\hline & & Observed & Effective & Observed $\left(\mathrm{H}_{0}\right)$ & Expected $\left(\mathrm{H}_{\mathrm{E}}\right)$ & & & \\
\hline Beagle & 82 & $5.3 \pm 2.7$ & $3.0 \pm 1.2$ & $0.587 \pm 0.122$ & $0.624 \pm 0.133$ & $0.567 \pm 0.149$ & $-0.045 \pm 0.174$ & * \\
\hline Bichon & 17 & $4.0 \pm 2.0$ & $2.5 \pm 1.1$ & $0.542 \pm 0.271$ & $0.531 \pm 0.232$ & $0.497 \pm 0.212$ & $-0.032 \pm 0.246$ & NS \\
\hline Schnauzer & 14 & $3.3 \pm 1.6$ & $2.1 \pm 0.9$ & $0.485 \pm 0.251$ & $0.480 \pm 0.206$ & $0.407 \pm 0.191$ & $-0.001 \pm 0.210$ & NS \\
\hline Total & 113 & $6.3 \pm 3.4$ & $3.6 \pm 1.6$ & $0.567 \pm 0.136$ & $0.662 \pm 0.162$ & $0.612 \pm 0.179$ & $-0.002 \pm 0.128$ & \\
\hline
\end{tabular}

MNA, mean number of alleles; PIC, polymorphism information content; $F_{I S}$, the measure of the deviation from the Hardy-Weinberg proportions within subpopulations; HWE, Hardy-Weinberg equilibrium; * $p<0.01$; NS, not significant.

Hardy-Weinberg equilibrium $(\mathrm{p}<0.01)$ (Table 4) in comparison to the other two populations.

\section{Inter-population genetic variation}

To estimate genetic variation among the five dog populations, two parameters $-F_{\mathrm{ST}}$ and genetic distance (D) calculated by the gene frequency of each animal at each microsatellite locus-were evaluated in this study. The values of $F_{\mathrm{ST}}$ and $\mathrm{D}$ for each test population pair are summarized in Table 5 . The $F_{\mathrm{ST}}$ for each population pair was highly significant $(\mathrm{p}<$ 0.05 ). The $F_{\mathrm{ST}}$ values of the population pairs varied from 0.165 (for the BET and BEJB population pair) to 0.405 (for the SC and BEJB population pair). The genetic distances between the dog population pairs varied from 0.400 (for the BET and BEJB population pair) to 0.993 (for the SC and BEJA population pair). Surprisingly, the second relatively low genetic distance and $F_{\mathrm{ST}}$ value were not between other $\mathrm{BE}$ populations. The genetic distances and $F_{\mathrm{ST}}$ values of the BI-BET and SC-BET population pairs were less than the BEJA-BEJB and BEJA-BET population pairs.

\section{Population differentiation analysis}

The Nei's standard genetic distance of these three populations of dogs in Taiwan was calculated. $\mathrm{A}_{\mathrm{A}}$ distance matrix was used to build an individual phylogenetic tree with the NJ method (Figure 1). The results showed that the individual phylogenetic tree could be divided into three main clusters: SC, BI, and BE. Among these, the BE cluster could be further divided into a Taiwan population (BET) and two Japanese sub-populations: a Japan A (BEJA) and Japan B (BEJB) popu-

Table 5. Pair-wise estimates of breed differentiation $\left(F_{\mathrm{ST}}\right)$ (above the diagonal) and genetic distance (D) (below the diagonal) between each pair of the five dog populations

\begin{tabular}{lccccc}
\hline Population & BI & SC & BET & BEJA & BEJB \\
\hline BI & - & 0.274 & 0.207 & 0.306 & 0.281 \\
SC & 0.523 & - & 0.227 & 0.405 & 0.287 \\
BET & 0.461 & 0.491 & - & 0.274 & 0.165 \\
BEJA & 0.701 & 0.993 & 0.788 & - & 0.262 \\
BEJB & 0.689 & 0.608 & 0.400 & 0.585 & - \\
\hline
\end{tabular}

$F_{\mathrm{ST}}$ : Wright's F-statistics, among population differentiation estimates. $\mathrm{BI}$, bichon; SC, schnauzer; BET, beagle (Taiwan); BEJA, beagle (Japan A); BEJB, beagle (Japan B). lation. A phylogenetic tree using the NJ method with bootstrap resampling $(n=1,000)$ of the 14 microsatellite loci was constructed with the PHYLIP software. In the NJ tree (Figure $2 \mathrm{~A}$ ), the entire dog population could also be divided into three main clusters: SC, BI, and BE. The results of the main clusters were consistent with the results of the individual phylogenetic tree (Figure 1). In addition, the bootstrap value between the SC and other dog populations was 100\%, which showed that the genetic distance between these groups was relatively large. Unlike the $\mathrm{NJ}$ method, the phylogenetic tree constructed by the unweighted arithmetic average pair group (UPGMA) method (Figure 2B) showed that the BEJA group was clearly distanced from others, with a bootstrap value of $100 \%$. The remaining dog groups were divided into three clusters: $\mathrm{SC}, \mathrm{BI}$, and BET and BEJB.

A PCoA of pair-wise genetic distances among the five examined dog sub-populations was used to represent the relative positions of the populations. The first (PC1), second (PC2), and third (PC3) PCos accounted for 44.15\%, 26.35\%, and $19.97 \%$ of the total variation, respectively (Figure 3 ). The distance between the BEJA and BEJB groups was relatively close. The distance between the BET group and both the $\mathrm{BEJA}$ and BEJB groups was closer than the distance between the BET group and both the SC and BI groups.

\section{Population structure analysis}

The STRUCTURE software program using Bayesian modelbased clustering algorithms of multi-locus genotypes was utilized to assign individuals to populations via estimated individual admixture proportions and to infer the number of populations $(\mathrm{K})$ for a given sample. The results of the analysis are shown in Figure 4. It was mainly divided into Taiwan Beagle population and other populations at $\mathrm{K}=2$. When $\mathrm{K}$ $=3$, the $\mathrm{BI}$ group was separated. At $\mathrm{K}=4$, it is mainly divided into BI, SC, BET, and BEJ four clusters. Until $\mathrm{K}=5$, the two $\mathrm{BRJ}$ clusters are separated finally. When the $\mathrm{K}$ value over 5 , the BET populations are further subdivided into different clusters.

\section{Probability of identity and power of exclusion}

The analysis of probability of identity involved three dog populations, respectively, and all populations together was 

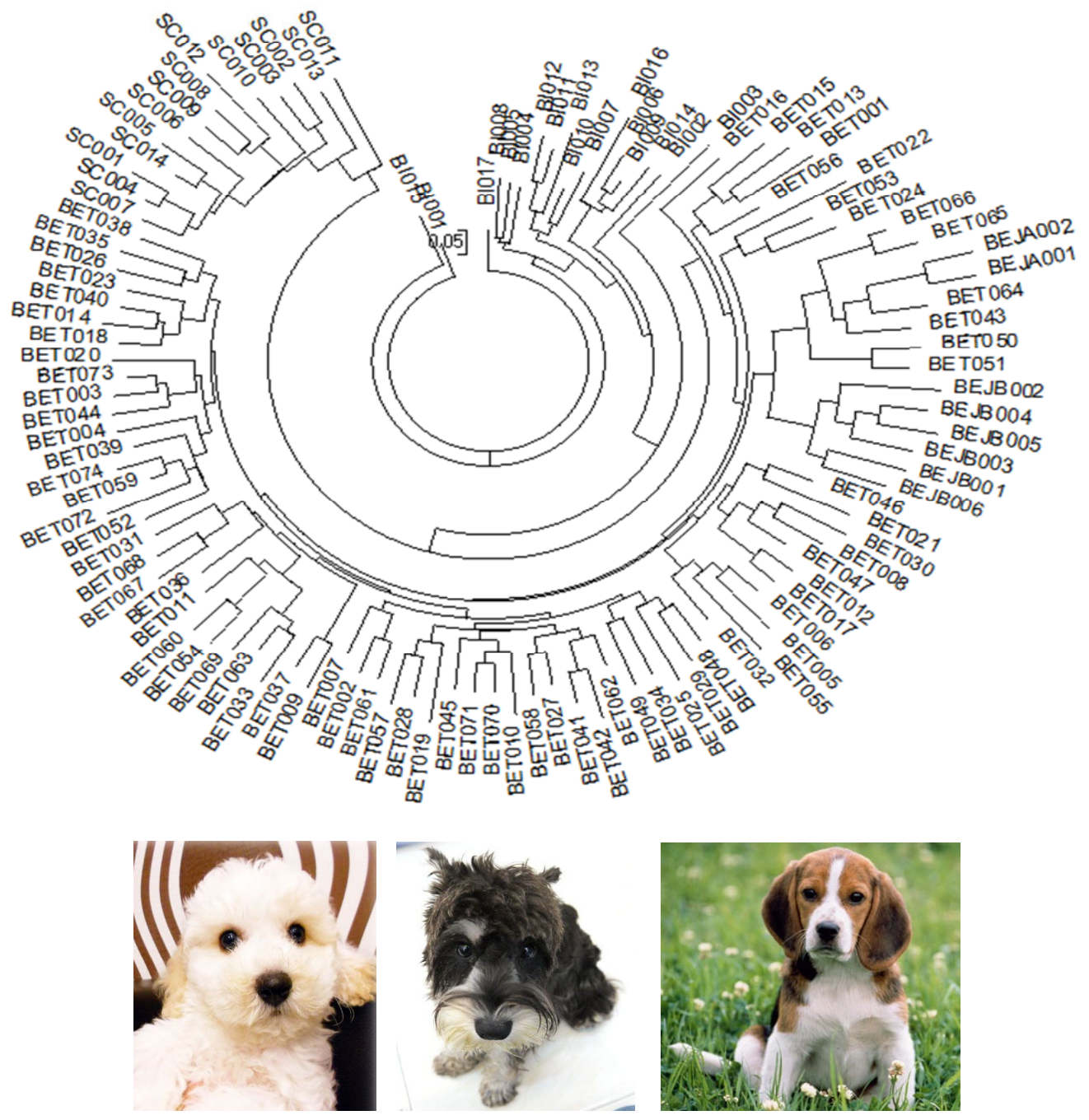

Figure 1. The neighbor-joining (NJ) tree among these dog individuals based on the 14 sets of novel microsatellite markers. SC, Schnauzer; $\mathrm{BI}$, Bichon; BET, Beagle (Taiwan); BEJA, Beagle (Japan A); BEJB, Beagle (Japan B) in this study.

(A)

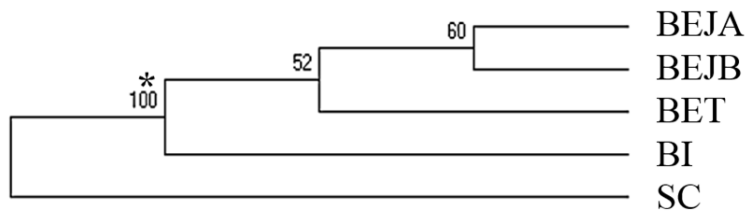

(B)

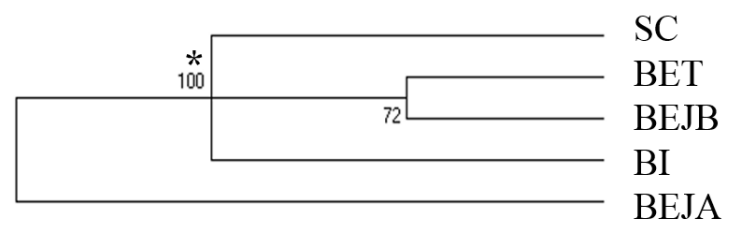

Figure 2. The $(A)$ neighbor-joining $(N J)$ tree and $(B)$ unweighted pair group method with arithmetic mean (UPGMA) tree among these dog populations based on the 14 sets of novel microsatellite markers. SC, Schnauzer; BI, Bichon; BET, Beagle (Taiwan); BEJA, Beagle (Japan A); BEJB, Beagle (Japan B). * Bootstrap values. 


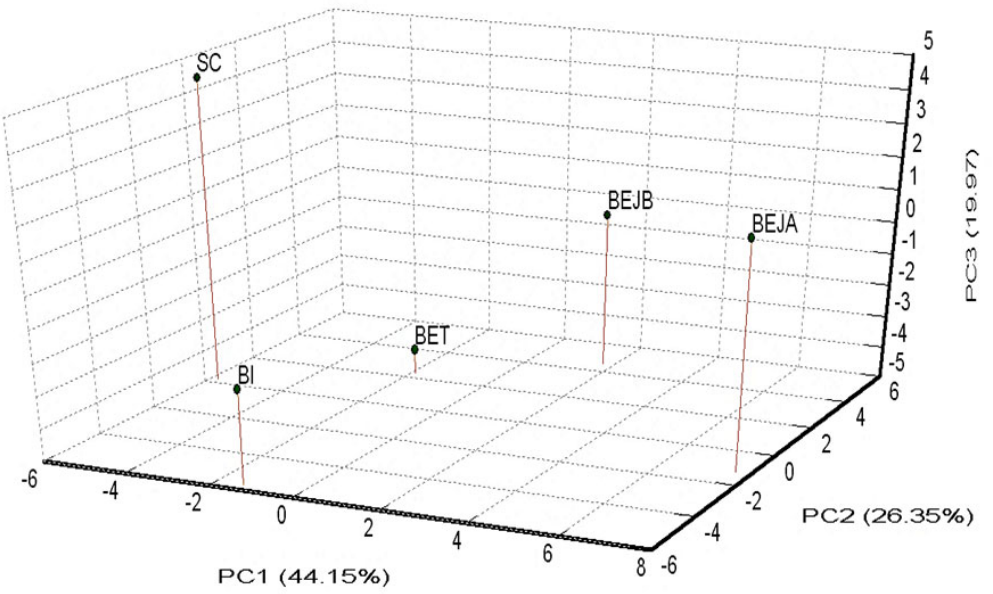

Figure 3. Principal coordinate analysis (PCoA) three-dimensional (3D) plot of dogs = relative to population genetic distances. SC, Schnauzer; $\mathrm{BI}$, Bichon; BET, Beagle (Taiwan); BEJA, Beagle (Japan A); BEJB, Beagle (Japan B).

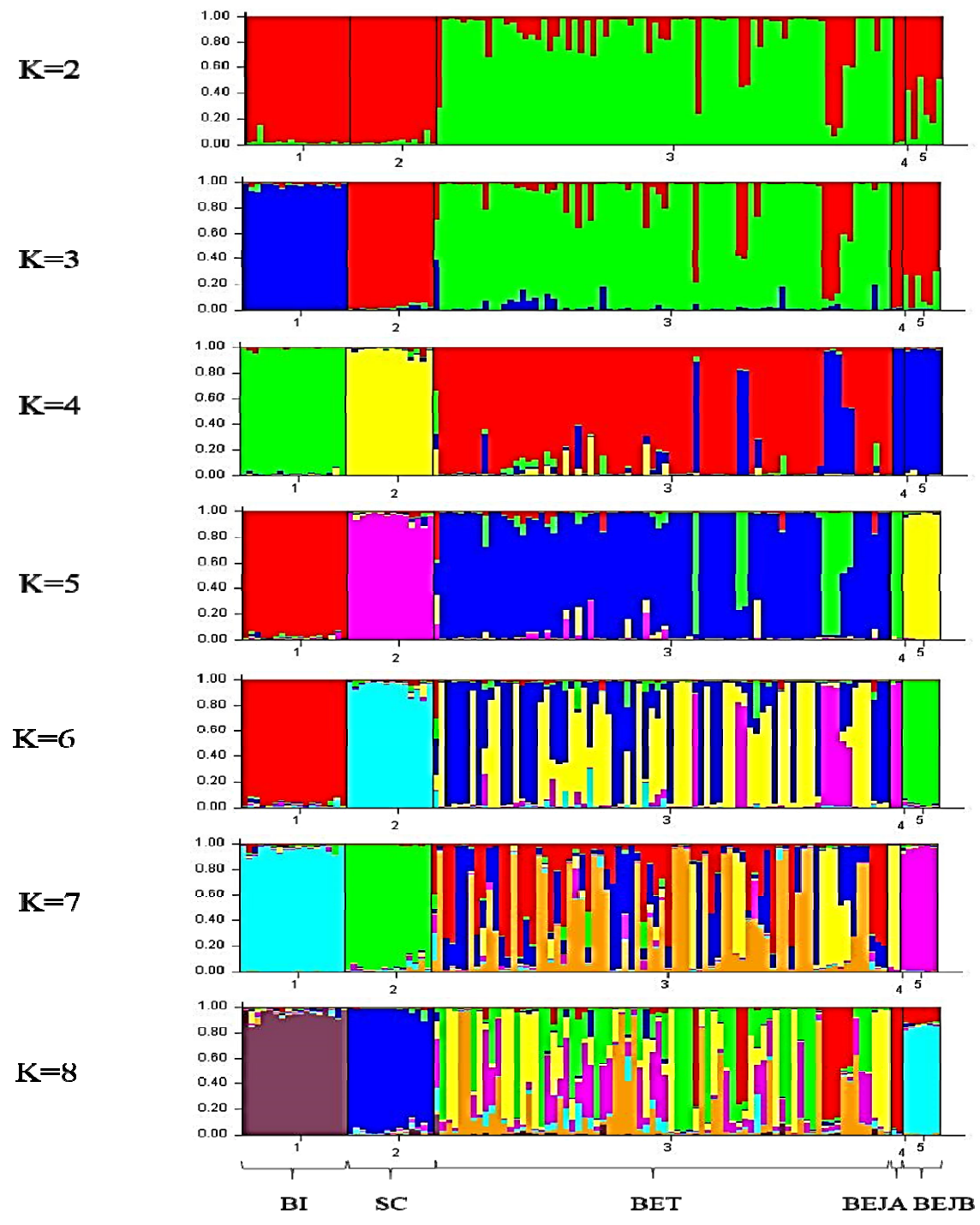

Figure 4. STRUCTURE cluster analysis plot $(K=2$ to 8$)$ of individual dogs based on the 14 sets of novel microsatellite markers. $K$ is the number of clusters assumed in the STRUCTURE analysis, and the colors corresponded to clusters. The vertical axis indicates the proportion of gene resources in individuals from the clusters, and each individual is represented by a single bar. SC, Schnauzer; BI, Bichon; BET, Beagle (Taiwan); BEJA, Beagle (Japan A); BEJB, Beagle (Japan B). 
calculated by the probability of identity $\left(\mathrm{P}_{(\mathrm{ID})}\right)$ and the identification rate of close relatives $\left(\mathrm{P}_{(\mathrm{ID}) \text { sib }}\right)$ of the 14 single new microsatellite markers. The results are listed in Table 6 and Table 7. The $\mathrm{P}_{\text {(ID) }}$ of $\mathrm{BE}$ populations per locus varied from 0.042 (SEL005) to 0.491 (SEL094). The combined $\mathrm{P}_{(\mathrm{ID})}$ values for all loci was $3.7 \times 10^{-11}$. The $\mathrm{P}_{(\mathrm{ID})}$ of each locus in BIs ranged from 0.060 (SEL005) to 1.000 (SEL094). The combined $P_{(\text {ID) }}$ values for all loci was $7.8 \times 10^{-9}$. The $\mathrm{P}_{\text {(ID) }}$ of SC populations per locus varied from 0.097 (SEL005) to 0.869 (SEL115). The combined $\mathrm{P}_{(\mathrm{ID})}$ values for all loci was $1.2 \times 10^{-7}$. In addition, the $\mathrm{P}_{\text {(ID) }}$ of the 14 novel microsatellite loci for all dog populations ranged from 0.031 (SEL005) to 0.843 (SEL094), and the comprehensive $\mathrm{P}_{(\mathrm{ID})}$ was $1.7 \times 10^{-12}$.

When individuals in the population to be explored have close relatives, such as full-sib or half-sib, it is more appropriate to evaluate the proportion of identification using the $\mathrm{P}_{(\mathrm{ID}) \mathrm{sib}}$. The $\mathrm{P}_{(\mathrm{ID}) \text { sib }}$ of $\mathrm{BE}$ populations per locus varied from 0.337 (SEL005) to 0.701 (SEL094). The combined $\mathrm{P}_{\text {(ID)sib }}$ values for all loci was $3.9 \times 10^{-5}$. In BIs, the $\mathrm{P}_{(\mathrm{ID}) \text { sib }}$ ranged from 0.358 (SEL005) to 1.000 (SEL094), and the combined $\mathrm{P}_{\text {(ID)sib }}$ values for all loci was $2.6 \times 10^{-4}$. The $\mathrm{P}_{(\mathrm{ID}) \text { sib }}$ of SC populations per locus varied from 0.403 (SEL005) to 0.933 (SEL115). The combined $\mathrm{P}_{(\mathrm{ID}) \text { sib }}$ values of SC for all loci was $7.1 \times 10^{-4}$.

In the paternity test for dogs, according to the formula of power of exclusion (PE) proposed by Jamieson [25], the value calculated using the 14 new microsatellite markers' allele frequencies was $99.98 \%$. This meant that when the genotypes of the mother and offspring were known, the possibility of being a biological father could be almost completely eliminated for an individual who was not a sire.

\section{DISCUSSION}

Table 6 . The probability of identity $\left(P_{(\mathrm{ID})}\right)$ of 14 sets of novel microsatellite markers in different dog breeds and the total dog population

\begin{tabular}{lcccc}
\hline Locus & Beagle & Bichon & Schnauzer & $\begin{array}{c}\text { Total } \\
\text { population }\end{array}$ \\
\hline SEL005 & 0.042 & 0.060 & 0.097 & 0.031 \\
SEL025 & 0.149 & 0.161 & 0.250 & 0.083 \\
SEL030 & 0.143 & 0.264 & 0.342 & 0.085 \\
SEL031 & 0.164 & 0.277 & 0.578 & 0.128 \\
SEL034 & 0.240 & 0.323 & 0.461 & 0.264 \\
SEL035 & 0.116 & 0.188 & 0.113 & 0.054 \\
SEL068 & 0.222 & 0.163 & 0.211 & 0.164 \\
SEL093 & 0.441 & 0.717 & 0.387 & 0.432 \\
SEL094 & 0.491 & 1.000 & 0.600 & 0.843 \\
SEL098 & 0.170 & 0.593 & 0.149 & 0.110 \\
SEL105 & 0.286 & 0.191 & 0.343 & 0.263 \\
SEL115 & 0.094 & 0.158 & 0.869 & 0.091 \\
SEL117 & 0.298 & 0.248 & 0.497 & 0.275 \\
SEL118 & 0.137 & 0.353 & 0.434 & 0.101 \\
Combined & $3.7 \times 10^{-11}$ & $7.8 \times 10^{-9}$ & $1.2 \times 10^{-7}$ & $1.7 \times 10^{-12}$ \\
\hline
\end{tabular}

$P_{(I D)}$, probability of identity.
Table 7. The probability of identity among sibs $\left(P_{(\mathbb{I D}) \text { sib }}\right)$ of 14 sets of novel microsatellite markers in different dog breeds and the total dog population

\begin{tabular}{lcccc}
\hline Locus & Beagle & Bichon & Schnauzer & $\begin{array}{c}\text { Total } \\
\text { population }\end{array}$ \\
\hline SEL005 & 0.337 & 0.358 & 0.403 & 0.323 \\
SEL025 & 0.444 & 0.451 & 0.533 & 0.385 \\
SEL030 & 0.432 & 0.525 & 0.600 & 0.383 \\
SEL031 & 0.468 & 0.536 & 0.768 & 0.425 \\
SEL034 & 0.519 & 0.576 & 0.678 & 0.538 \\
SEL035 & 0.428 & 0.481 & 0.412 & 0.359 \\
SEL068 & 0.509 & 0.466 & 0.490 & 0.466 \\
SEL093 & 0.661 & 0.849 & 0.608 & 0.653 \\
SEL094 & 0.701 & 1.000 & 0.778 & 0.740 \\
SEL098 & 0.472 & 0.773 & 0.445 & 0.410 \\
SEL105 & 0.539 & 0.468 & 0.584 & 0.524 \\
SEL115 & 0.405 & 0.469 & 0.933 & 0.406 \\
SEL117 & 0.547 & 0.522 & 0.706 & 0.533 \\
SEL118 & 0.439 & 0.592 & 0.654 & 0.398 \\
Combined & $3.9 \times 10^{-5}$ & $2.6 \times 10^{-4}$ & $7.1 \times 10^{-4}$ & $1.6 \times 10^{-5}$ \\
\hline P(D)
\end{tabular}

$\mathrm{P}_{(\mathrm{I}) \text { sib, }}$ probability of identity among sibs.

In this study, 14 sets of novel microsatellite markers were developed and used to analyze the genetic variation of three dog breeds-SC, BI, and BE. The results showed that the Na of the 14 novel microsatellite loci was 6.3 , and the Ne was 3.6 (Table 3). The Na of locus SEL115 was 13, whereas the Ne was only 3.8. The reason might have been that the distribution of allele frequency was mainly concentrated on three alleles-243 bp (13.3\%), 247 bp (10.6\%), and 251 bp (47.4\%) (Supplementary Table S1) - such that in some cases the uneven distribution of allele frequencies caused a large gap between the $\mathrm{Na}$ and the $\mathrm{Ne}$.

The $\mathrm{H}_{\mathrm{E}}, \mathrm{H}_{\mathrm{O}}$, and PIC are commonly used to assess the polymorphism of microsatellite loci in the analyzed population. The $\mathrm{H}_{\mathrm{O}}$ refers to the observed heterozygosity of each locus, which represents the actual proportion of heterozygous individuals in the population. The $\mathrm{H}_{\mathrm{E}}$ is the expected heterozygosity of each locus, which is the expected proportion of heterozygous individuals in the population that is calculated according to the Hardy-Weinberg Law. The PIC is the degree of polymorphism of each locus. Using the 14 novel microsatellite markers to analyze our dog populations, the average $H_{E}$ was 0.662 , which showed that the values of most of the microsatellite markers were within high expected heterozygosity $\left(\mathrm{H}_{\mathrm{E}}>0.5\right)$. The average value of $\mathrm{H}_{\mathrm{O}}$ was 0.567 , which also fell within the range of high observed heterogeneity $\left(0.7>\mathrm{H}_{\mathrm{O}}>0.5\right)$ [26]. The average value of PIC was 0.612 , which fell within the range of high polymorphic information content (PIC>0.5) [27] (Table 3). The results of this experiment were similar to the study of Radko et al [28] using 18 sets of microsatellite markers to analyze the Polish Tatra shepherd dog. Their results showed that the average $\mathrm{H}_{\mathrm{E}}$ was 
0.643 , the average $\mathrm{H}_{\mathrm{O}}$ was 0.645 , the average PIC was 0.598 , and the values of the three variables were all greater than 0.5 . Therefore, their study indicated that the tested Tatra shepherd dog population was highly polymorphic. In another study [29], eight breeds of dog were surveyed by 21 microsatellite markers. PIC values over 0.5 were measured for 15 markers. The average value of the PIC was 0.555 . Compared with these reports, the three variables in the current experiment were highly polymorphic, indicating that the 14 novel microsatellite loci should be able to effectively analyze the genetic structure and genetic variation of the three breeds of dogs analyzed in this experiment.

The $\mathrm{H}_{\mathrm{E}}(0.412$ and 0.293$)$ and $\mathrm{H}_{\mathrm{O}}(0.451$ and 0.248$)$ values of loci SEL093 and SEL094 were both less than 0.5, as were the respective PIC ( 0.326 and 0.249$)$ values. The cause of this result, for which there were only two alleles in two loci, was supposed to be sampling error. However, some reports have suggested that the number of alleles for microsatellite markers should be three or more to reduce the standard deviation of distance calculation [30]. The reason why the loci SEL093 and SEL094 were selected in this experiment was that the number of dog breeds analyzed was relatively small. If the number of breeds is increased, perhaps the allele number of these two microsatellite loci could be increased, and the three variables will be likely to increase as well. On this basis, the two microsatellite loci SEL093 and SEL094 were retained as potential canine microsatellite loci in this experiment.

In terms of the analysis of population genetic structure, we applied the $F_{\mathrm{IS}}, F_{\mathrm{ST}}$ and $F_{\mathrm{IT}}$ statistics to evaluate the distribution of genetic variation within and between populations. The average $F_{\text {IS }}$ value of the 14 new microsatellite markers was 0.002 . This value was positive and low. The percentage of heterozygotes in the overall tested dog population was less than expected-that is, there was an inbreeding phenomenon-but the average value of $F_{\text {IS }}$ was around 0.002 , which indicated that the situation in the dog population was not serious. The mean $F_{\mathrm{ST}}$ for all the loci was 0.212 . This fell within the range of high differentiation $\left(0.15<F_{\mathrm{ST}}<0.25\right)$, according to the Sewall Wright rules [31], indicating that there was high differentiation among the three breeds of dogs in this study.

Kang et al [32] investigated the genetic structure of local dogs in South Korea and establish an individual and paternity identification system through evaluating the polymorphisms of the populations with three variables: $\mathrm{H}_{\mathrm{O}}, \mathrm{H}_{\mathrm{E}}$, and PIC. Between nine and 11 microsatellite loci were used for genetic analysis of two local breeds from South Korea and three exotic dog breeds in their study. The sample selection criterion was at least one generation of unrelated dog individuals. The results showed that the average $\mathrm{H}_{\mathrm{O}}$ for each breed ranged from 0.65 to 0.78 ; the $\mathrm{H}_{\mathrm{E}}$ ranged from 0.71 to 0.85 ; and the PIC ranged from 0.66 to 0.82 . In this study, the average value of $\mathrm{H}_{\mathrm{E}}$ for the three varieties ranged from 0.480 (SCs) to 0.624 (BEs), and average $\mathrm{H}_{\mathrm{O}}$ ranged from 0.485 (SCs) to 0.587 (BEs). The average PICs ranged between 0.407 (SCs) and 0.567 (BEs) (Table 4). Compared with the abovementioned studies, the three variables in our experiment showed slightly lower values, which may have been caused by differences in the individual dogs included in this experiment: some of the animals were blood-relatives and were full-sibs or half-sibs, so their genetic backgrounds were similar, leading to slightly lower polymorphisms. Future testing of individual Taiwanese dogs with different origins or more distant blood relationships could improve the applicability of these new microsatellite markers.

Among the different varieties of $F_{\text {IS }}$, only the average value for BEs (0.045) was positive. The results showed that although BEs in this experiment had high genetic variation, the positive value of $F_{\text {IS }}$ indicated that the proportion of heterozygous individuals was still too small to achieve the Hardy-Weinberg balance; Iindeed, it deviated significantly from the expected value $(\mathrm{p}<0.01)$. This result may reflect the fact that the fathers of the BE population in this experiment comprised a small number of male dogs, which was not reflective of the situation of mating by chance. The BIs and SCs did not deviate from the Hardy-Weinberg equilibrium, and the $F_{\text {IS }}$ values of these two breeds were negative. It can thus be inferred that these two breeds had no inbreeding issues, the genetic backgrounds of the parents were different, and the number of male and female animals was equal [33]. Therefore, it was supposed that the deviation of the entire dog population from the Hardy-Weinberg balance was attributable to the deviation of the BE population.

The individual phylogenetic tree of the dogs (Figure 1) was constructed by the NJ method, and the cluster analysis diagram drawn by the STRUCTURE software (Figure 4), and the dogs in this experiment were divided into five groups: SC, BI, BET, BEJA, and BEJB. In the phylogenetic tree of dog populations drawn by the NJ method (Figure $2 \mathrm{~A}$ ), the distinctive main clusters were consistent with the result of the individual phylogenetic tree: both could clearly distinguish the $\mathrm{BI}, \mathrm{SC}$, and $\mathrm{BE}$ groups, and the BET and BEJ groups were closely identified. The different breeds of dogs could be clearly differentiated by the microsatellite markers used in this experiment. The differentiation of dog breed in tree would be caused by the unique alleles. For example, the alleles $185 \mathrm{bp}$ and $201 \mathrm{bp}$ of the SEL035 locus were only found in the BI and SC populations, respectively (Supplementary Table S1). In the phylogenetic tree drawn by the NJ method, the bootstrap value of the BET and the BEJ group was $52 \%$, which means that only $52 \%$ of the analysis results separated them. When the bootstrap value between the two populations was not greater than $70 \%$ in neighbor joining tree, it showed that the clustering of that two populations were not obvious [34]. 
In the NJ phylogenetic tree, the bootstrap value between the SC and other dog populations was $100 \%$, and the result was in the highly reliable range (bootstrap value $>70 \%$ ). That showed that the genetic distance between the SC and other dog populations was relatively long. However, the UPGMA phylogenetic tree (Figure $2 \mathrm{~B}$ ) shows that the BEJA group was far away from other dog groups, and the bootstrap value was $100 \%$. But the UPGMA method based on constant-rate assumption, and the distance between samples on the same branch is the same, so this method is only suitable for the case where all samples have the same evolution distance [35], so recently it was less used to construct a phylogenetic tree, and the NJ method was a more commonly used method for drawing a phylogenetic tree.

The PCoA draws a 3D stereogram based on the genetic distance between the populations (Figure 3), and distinguishes the genetic distance among the populations by the variance of the three principal coordinate axes. It can be found that the two BEJ groups were relatively close, which was consistent with the close geographical relationship between the two, and the relative distance between the two groups and the BET group was closer than the relative distance between the two groups and the SC and the BI. The results of the NJ phylogenetic tree can also be confirmed in the 3D map of the PCoA. The SC and the BEJA were located on the farthest sides of the 3D map, so there was a maximum genetic distance between the two populations.

In the individual analysis, the combined probability of identification $\left(\mathrm{P}_{(\mathrm{ID})}\right)$ of the 14 new microsatellite loci for the entire dog population was $1.7 \times 10^{-12}$ (Table 6), and according to the survey of the Council of Agriculture up until the end of 2019 , the total number of dogs in Taiwan was about $1.54 \times 10^{6}$ [36]. That meant when the current number of Taiwanese dog populations is analyzed by the 14 new microsatellite markers used in this experiment, the probability of appearing exactly the same genotype is very low. Although the dog population in this experiment contained only three breeds, however, when examining $\mathrm{P}_{(\mathrm{ID})}$ of a single dog breed, the credibility is still high. There are no statistics on the numbers of dogs of different breeds currently in Taiwan; however, it can be ascertained that the number of dogs in any single breed cannot exceed the total number of dogs. Therefore, the $\mathrm{P}_{\text {(ID) }}$ of the three dog populations respectively in this study should cover the total number of dogs in Taiwan in 2019. It was confirmed that the probability of the same genotype being identified in two individuals with 14 the sets of novel microsatellite markers was very low.

The combined probability of identity among sibs $\left(\mathrm{P}_{(\mathrm{ID}) \text { sib }}\right)$ was $1.6 \times 10^{-5}$ (Table 7) in this study. According to Waits et al [24], their markers were sufficient to identify close relatives of the natural population when the $\mathrm{P}_{(\mathrm{ID}) \text { sib }}$ was between $10^{-3}$ and $10^{-4}$. The $\mathrm{P}_{(\mathrm{ID}) \text { sib }}$ values of the three breeds of dogs in this experiment generally met this recommended standard. This confirmed that the 14 novel microsatellite markers were applicable in the individual identification of close relatives of BEs, BIs, and SCs in Taiwan.

In the paternity tests of dogs, the PE of the 14 new microsatellite markers in this experiment was $99.98 \%$, which meant that when the genotypes of the mother and offspring were known, individuals that were not the biological father could be ruled out. According to the pedigree data for the BE population collected in this experiment, there was one of the cases where the mother-child genotypes were known, the individual registered as the biological father in the pedigree was compared with the genotypes of 14 novel microsatellite markers and which was not found to provid any of the alleles to the offspring at six of the loci. Therefore, it could be inferred that the male was not the biological father, and suggested that the registration of the dog's pedigree data was inaccurate. This supports the suggestion that many dog-breeding facilities in Taiwan are still not rigorous enough for pedigree registration. Therefore, using paternity facilities with high $\mathrm{PE}$ to improve the registration of pedigree in Taiwan is important. The microsatellite markers developed in this study may be suitable for this purpose to avoid the potential damage caused by inbreeding.

At present study, many countries used microsatellite markers to analyze dog populations. For developing a platform of paternity and individual identification, the American Kennel Club (AKC) analyzed 108 dog breeds using 17 microsatellite markers. The results show that the average $\mathrm{H}_{\mathrm{O}}$ was 0.60 , and the average value of PIC was 0.56 . PE was more than $99 \%$ in all breeds, and the combined $\mathrm{P}_{(\mathrm{ID})}$ was $3.2 \times 10^{-8}$. The American Kennel Association considered that 17 microsatellite markers were sufficient for ordinarily paternity identification [37]. Eichmann et al. established Austrian dog DNA profiling for investigation of dog-related accidents and crimes, using 15 sets of highly polymorphic microsatellite markers to analyze $45 \mathrm{dog}$ breeds [38]. The results revealed that the average $\mathrm{H}_{\mathrm{O}}$ was 0.74 , the average PIC was 0.82 , and the combined $\mathrm{P}_{(\mathrm{ID}) \text { sib }}$ was $8.5 \times 10^{-8}$, showing that these 15 microsatellite markers are sufficient for individual identification of dogs in Austria. Kang et al [32] used 9 to 11 sets of microsatellite markers to detect the genetic structure of local dogs in South Korea and established an individual and paternity identification system to perform genetic analysis of two local breeds of South Korea and three foreign dog breeds. The results showed that the average $\mathrm{H}_{\mathrm{O}}$ for each breed was between 0.65 and 0.78 ; the average PIC was between 0.66 and 0.82 ; and the average $\mathrm{PE}$ was more than $99 \%$ in all breeds. The average $\mathrm{H}_{\mathrm{O}}$ value $(0.57)$ of the 14 new microsatellite markers in this study near the range of the research results of the previous countries ( 0.60 to 0.74 ). The average value of PIC (0.61) was also within the range 
of the results of the aforementioned countries (0.56 to 0.82). According to the researches before, the 14 new microsatellite markers developed in this study were highly polymorphic and suitable to analyze the three breeds of dogs in Taiwan.

In conclusion, using 14 novel microsatellite markers to analyze the beagle, bichon, and schnauzer populations in Taiwan, the results showed that their average expected heterozygosity, observation heterozygosity, and polymorphism information content were all at high levels. Therefore, these new microsatellite markers have high applicability to the analyzed populations. These results indicate that the new microsatellite markers have good resolution when applied to the detection of differences among dog breeds. It was confirmed that the opportunity of identifying the exact same genotype among the analysis of the 14 new microsatellite markers was very low. In addition, the power exclusion was enough high to be a good tool for paternity testing.

\section{CONFLICT OF INTEREST}

We certify that there is no conflict of interest with any financial organization regarding the material discussed in the manuscript.

\section{FUNDING}

This work was financially supported by the Council of Agriculture, Executive Yuan, Taiwan (Grant No.: 2012 AS-2.1.9AD-U1 and 2013 AS-2.1.9-AD-U1) in Taiwan.We would like to acknowledge the National Center for Genome Medicine (NCGM), National Science Council, Taiwan, for their technical support with microsatellite markers.

\section{REFERENCES}

1. Streitberger K, Schweizer M, Kropatsch R, et al. Rapid genetic diversification within dog breeds as evidenced by a case study on Schnauzers. Anim Genet 2012;43:577-86. https:// doi.org/10.1111/j.1365-2052.2011.02300.x

2. Whitehouse-Tedd K, Richards N, Parker M. Dogs and Conservation: emerging themes and considerations. J Vertebr Biol 2021;69:E2004.1-4. https://doi.org/10.25225/jvb.E2004

3. Ottesen JL, Weber A, Gürtler H, Mikkelsen LF. New housing conditions: improving the welfare of experimental animals. Altern Lab Anim 2004;32(Suppl 1B):397-404. https://doi. org/10.1177/026119290403201s65

4. Lust G. An overview of the pathogenesis of canine hip dysplasia. J Am Vet Med Assoc 1997;210:1443-5.

5. Rooney N. Sargan D. Pedigree dog breeding in the UK: A major welfare concern? [cited 2021 July 19]. Horsham, UK: Royal Society for the Prevention of Cruelty to Animals; 2009. Available from: https://www.rspca.org.uk/webContent/static
Images/Downloads/PedigreeDogsReport.pdf

6. Chia R, Achilli F, Festing MFW, Fisher EMC. The origins and uses of mouse outbred stocks. Nat Genet 2005;37:11816. https://doi.org/10.1038/ng1665

7. The Kennel Club. Getting a dog. [cited 2021 July 21]. Available from: http://www.thekennelclub.org.uk

8. Lindblad-Toh K, Wade CM, Mikkelsen TS, et al. Genome sequence, comparative analysis and haplotype structure of the domestic dog. Nature 2005;438:803-19. https://doi.org/ 10.1038/nature04338

9. Ostrander EA, Mapa FA, Yee M, Rine J. One hundred and one new simple sequence repeat-based markers for the canine genome. Mamm Genome 1995;6:192-5. https://doi. org/10.1007/BF00293011

10. Dvorak J, Halverson J, Stevenson T. Microsatellite sequences for canine genotyping. Washington, DC, USA: U.S. Patent and Trademark Office; 1999. U.S. Patent No. 5874217.

11. Ichikawa Y, Takagi K, Tsumagari S, et al. Canine parentage testing based on microsatellite polymorphism. J Vet Med Sci 2001;63:1209-13. https://doi.org/10.1292/jvms.63.1209

12. Irion DN, Schaffer AL, Famula TR, Eggleston ML, Hughes SS, Pedersen NC. Analysis of genetic variation in $28 \mathrm{dog}$ breed populations with 100 microsatellite markers. J Hered 2003;94:81-7. https://doi.org/10.1093/jhered/esg004

13. Wictum E, Kun T, Lindquist C, Malvick J, Vankan D, Sacks B. Developmental validation of DogFiler, a novel multiplex for canine DNA profiling in forensic casework. Forensic Sci Int Genet 2013;7:82-91. https://doi.org/10.1016/j.fsigen.2012. 07.001

14. van Hooft WF, Hanotte O, Wenink PW, et al. Applicability of bovine microsatellite markers for population genetic studies on African buffalo (Syncerus caffer). Anim Genet 1999;30: 214-20. https://doi.org/10.1046/j.1365-2052.1999.00453.x

15. Glenn TC, Schable NA. Isolating microsatellite DNA loci. Methods Enzymol 2005;395:202-22. https://doi.org/10.1016/ S0076-6879(05)95013-1

16. Schuelke M. An economic method for the fluorescent labeling of PCR fragments. Nat Biotechnol 2000;18:233-4. https://doi. org/10.1038/72708

17. Raymond M, Rousset F. GENEPOP (version 1.2): population genetics software for exact tests and ecumenicism. J Hered 1995;86:248-9. https://doi.org/10.1093/oxfordjournals.jhered. a111573

18. Saitou N, Nei M. The neighbor-joining method: a new method for reconstructing phylogenetic trees. Mol Biol Evol 1987;4: 406-25. https://doi.org/10.1093/oxfordjournals.molbev. a040454

19. Dieringer D, Schlotterer C. Microsatellite analyser (MSA): a platform independent analysis tool for large microsatellite data sets. Mol Ecol 2003;3:167-9. https://doi.org/10.1046/ j.1471-8286.2003.00351.x

20. Felsenstein J. Phylogeny Inference Package (PHYLIP) [Inter- 
net]. Genomes Sciences, Department of Genetics, Seattle, WA, USA: Washington University; 2002. Available from: http://evolution.gs.washington.edu/phylip.html

21. Felsenstein J. Confidence limits on phylogenies: an approach using the bootstrap. Evolution 1985;39:783-91. https://doi. org/10.1111/j.1558-5646.1985.tb00420.x

22. Pritchard JK, Stephens M, Donnelly P. Inference of population structure using multilocus genotype data. Genetics 2000;155: 945-59. https://doi.org/10.1093/genetics/155.2.945

23. Peakall R, Smouse PE. GenAlEx ver6.5: genetic analysis in Excel. Population genetic software for teaching and research - an update. Bioinformatics 2012;28:2537-9. https://doi.org/ 10.1093/bioinformatics/bts460

24. Waits LP, Luikart G, Taberlet P. Estimating the probability of identity among genotypes in natural populations: cautions and guidelines. Mol Ecol 2001;10:249-56. https://doi.org/10. 1046/j.1365-294X.2001.01185.x

25. Jamieson A. The genetics of transferrins in cattle. Heredity 1965;20:419-41. https://doi.org/10.1038/hdy.1965.54

26. Fonteque GV, Battilana J, Paludo E, Lima-Rosa CADV. Genetic polymorphism of fifteen microsatellite loci in Brazilian (blueegg Caipira) chickens. Pesq Vet Bras 2014;34:98-102. https:// doi.org/10.1590/S0100-736X2014000100016

27. Agha SH, Pilla F, Galal S, et al. Genetic diversity in Egyptian and Italian goat breeds measured with microsatellite polymorphism. J Anim Breed Genet 2008;125:194-200. https:// doi.org/10.1111/j.1439-0388.2008.00730.x

28. Radko A, Rubiś D, Szumiec A. Analysis of microsatellite DNA polymorphism in the Tatra Shepherd Dog. J Appl Anim Res 2018;46:254-6. https://doi.org/10.1080/09712119.2017.1292 912

29. Radko A, Podbielska A. Microsatellite dna analysis of genetic diversity and parentage testing in the popular dog breeds in Poland. Genes 2021;12:485. https://doi.org/10.3390/genes

\section{5}

30. Barker JSF. A global protocol for determining genetic distances among domestic livestock breeds. Proc 5th World Congress on Genetics Applied to Livestock Production; 1994. Vol. 21 pp. 501-8.

31. Wright S. Evolution and genetics of populations. Chicago, IL, USA: University of Chicago; 1978.

32. Kang BT, Kim KS, Min MS, et al. Microsatellite loci analysis for the genetic variability and the parentage test of five dog breeds in South Korea. Genes Genet Syst 2009;84:245-51. https://doi.org/10.1266/ggs.84.245

33. Hau J, Schapiro SJ. Handbook of laboratory animal science. In: Festing MFW, Lutz C, editors. Laboratory animal genetics and genetic quality control. 3rd ed. Boca, Raton, FL, USA: CRC Press; 2010. pp. 209-50.

34. Efron B, Halloran E, Holmes S. Bootstrap confidence levels for phylogenetic trees. Proc Natl Acad Sci (USA) 1996;93: 7085-90. https://doi.org/10.1073/pnas.93.14.7085

35. Barry GH. Phylogenetic trees made easy: a how-to manual. 3rd ed., Sunderland, MA, USA: Sinauer Assoc. Inc., 2007.

36. Animal Protection Information Network. Council of Agriculture, Executive Yuan c2019 [cited 2021 July 22]. Available from: https://animal.coa.gov.tw/Frontend/Know/Detail/ LT00000559? parentID=Tab0000004

37. DeNise S, Johnston E, Halverson J, et al. Power of exclusion for parentage verification and probability of match for identity in American Kennel Club breeds using 17 canine microsatellite markers. Anim Genet 2004;35:14-7. https://doi.org/10.1046/j. 1365-2052.2003.01074.x

38. Eichmann C, Berger B, Steinlechner M, Parson W. Estimating the probability of identity in a random dog population using 15 highly polymorphic canine STR markers. Forensic Sci Int 2005;151:37-44. https://doi.org/10.1016/j.forsciint.2004. 07.002 